\title{
A self-consistent renormalized jellium approach for calculating structural and thermodynamic properties of charge stabilized colloidal suspensions
}

Cite as: J. Chem. Phys. 131, 074115 (2009); https://doi.org/10.1063/1.3211305

Submitted: 08 May 2009. Accepted: 03 August 2009 . Published Online: 21 August 2009

Thiago E. Colla, Yan Levin, and Emmanuel Trizac

\section{ARTICLES YOU MAY BE INTERESTED IN}

Ionic size effects on the Poisson-Boltzmann theory

The Journal of Chemical Physics 147, 014104 (2017); https://doi.org/10.1063/1.4990737

Equation of state of charged colloidal suspensions and its dependence on the thermodynamic route

The Journal of Chemical Physics 136, 194103 (2012); https://doi.org/10.1063/1.4718367

Colloidal charge renormalization in suspensions containing multivalent electrolyte

The Journal of Chemical Physics 132, 104105 (2010); https://doi.org/10.1063/1.3354120

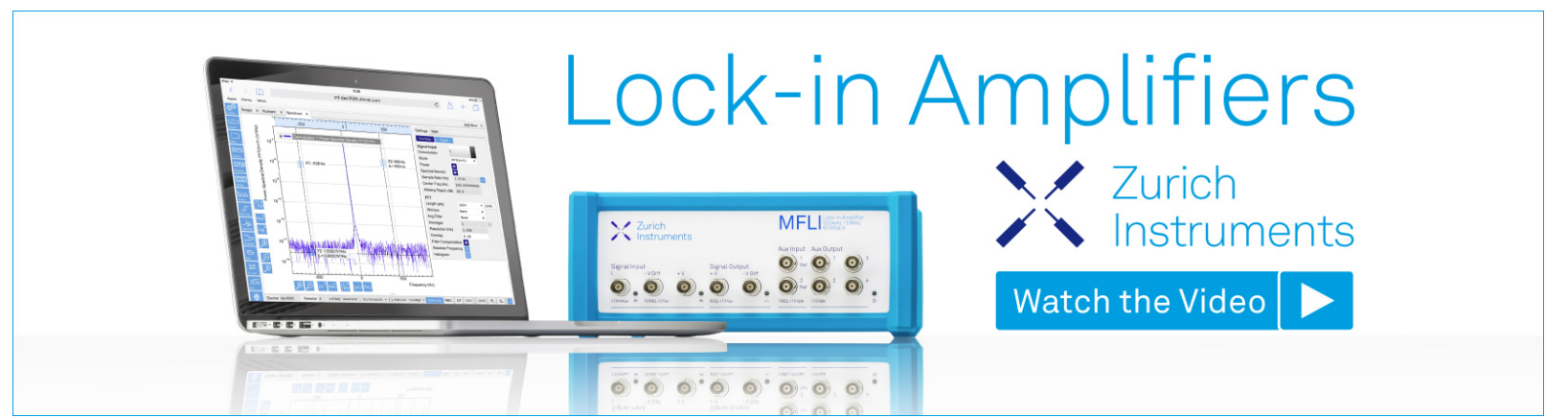

J. Chem. Phys. 131, 074115 (2009); https://doi.org/10.1063/1.3211305 


\title{
A self-consistent renormalized jellium approach for calculating structural and thermodynamic properties of charge stabilized colloidal suspensions
}

\author{
Thiago E. Colla, ${ }^{1, a)}$ Yan Levin, ${ }^{1, b)}$ and Emmanuel Trizac ${ }^{2, c)}$ \\ ${ }^{1}$ Instituto de Física, Universidade Fedaral do Rio Grande do Sul, CP 15051, 91501-970 Porto Alegre, \\ RS, Brazil \\ ${ }^{2}$ CNRS, Université Paris-Sud, UMR 8626, LPTMS, F-91405 Orsay Cedex, France
}

(Received 8 May 2009; accepted 3 August 2009; published online 21 August 2009)

\begin{abstract}
An approach is proposed which allows to self-consistently calculate the structural and the thermodynamic properties of highly charged aqueous colloidal suspensions. The method is based on the renormalized jellium model with the background charge distribution related to the colloid-colloid correlation function. The theory is used to calculate the correlation functions and the effective colloidal charges for suspensions containing additional monovalent electrolyte. The predictions of the theory are in excellent agreement with Monte Carlo simulations. (C) 2009 American Institute of Physics. [DOI: 10.1063/1.3211305]
\end{abstract}

\section{INTRODUCTION}

Over the span of the last century, colloidal suspensions have been the subject of intense theoretical and experimental study. The great effort is well justified by the importance that these systems play in industrial, biological, and medical applications. A practical problem that arises is how to stabilize suspensions against flocculation and precipitation, resulting from the short range attractive van der Waals interaction. One approach is to synthesize colloidal particles with acidic groups on their surface. In aqueous environment, these groups become ionized, resulting in a repulsion between the macroions.

Charge stabilized colloidal suspensions are an extreme example of a large asymmetry electrolyte. Both the charge and the size of the macroions are orders of magnitude larger than those of other ionic species present inside the suspension. Typically a colloidal particle of radius $1000 \AA$ will carry $10^{3}-10^{4}$ ionizable groups uniformly distributed over its surface. The huge asymmetry between the macroions and the microions makes the theoretical investigation of colloidal suspensions a very difficult task. ${ }^{1-4}$ The standard approach used to study these systems is based on the primitive model $(\mathrm{PM})$, which treats solvent as a dielectric continuum of permittivity $\epsilon$. The interaction potential between the ionic species is taken to be composed of a long range Coulomb interaction and a short range hard-core repulsion. Unfortunately, due to the large charge and size asymmetry between the macroions and the microions, even for this simplified model, the traditional methods of liquid state theory-such as the molecular dynamics simulations, Monte Carlo (MC) simulations, and the integral equations theories-prove to be only partially successful. ${ }^{2}$ The integral equations are plagued by the convergence problems, ${ }^{5}$ while the huge number of coun-

\footnotetext{
${ }^{a)}$ Electronic mail: colla@if.ufrgs.br.

${ }^{b}$ Electronic mail: levin@if.ufrgs.br.

${ }^{c)}$ Electronic mail: trizac@1ptms.u-psud.fr.
}

terions needed to ensure the bulk electroneutrality allows the maximum charge asymmetry, which can be accurately simulated using the present day computers to be around 100:1. The important case of added electrolyte remains practically unaccessible to direct computer simulations. Nevertheless, simulations have been extensively used to describe dynamical, thermodynamic, as well as structural properties of charged colloidal suspensions. ${ }^{6}$

To obtain a more tractable description of these systems, it is, therefore, necessary to introduce further simplifications. This can be achieved by integrating out the microion degrees of freedom, leaving only a state dependent interaction potential between the colloidal particles. This defines the, so called, one component model (OCM). In spite of its apparent simplicity, the OCM requires knowledge of the effective macroion-macroion interaction, which implicitly depends on all the ionic species. Formally, the potential can be obtained by explicitly tracing out the degrees of freedom of the microions of the PM. ${ }^{2,7}$ This can, in principle, be achieved using MC simulations. ${ }^{8}$ However, such approach is time consuming and is not always practical. A more direct method is to use approximate theories, such as the Poisson Boltzmann $^{7,9}$ or the Ornstein-Zernike (OZ) equations, with appropriate closure relations. ${ }^{5,10,11}$ Furthermore, to avoid computational difficulties, one usually assumes that the effective interaction potential is pairwise additive. This is quite reasonable at low macroion concentrations, however, care must be used when applying this assumption to more concentrated systems. As the concentration increases, the manybody correlations start to play an important role for both structural and thermodynamic properties. Assuming the OCM description with pairwise macroion interactions, there still remains a question of how to obtain the effective interaction potential. This has been the subject of many works. ${ }^{1,12-14}$ The difficulty in answering it is due to various factors, among which are strong correlations between the various particles and the huge asymmetry between the differ- 
ent ionic species-forcing different approximations for different correlations. In the limit of large dilutions, small colloidal charge, and monovalent electrolyte, a linearized Debye-Hückel theory can be applied and the pair potential takes a simple Yukawa-like form, known as the DerjaguinLandau-Overbeek-Verwey (DLVO) potential. For a system of colloidal particles of radius $a$, charge $-Z_{o} q$, density $\rho_{o}$, and microions of valence $z_{i}$ and bulk concentrations $\rho_{i}, i>0$, the DLVO potential is given by

$$
\beta u(r)=\ell_{b}\left(\frac{Z_{o}}{(1+\kappa a)}\right)^{2} \frac{e^{-\kappa(r-2 a)}}{r},
$$

where $\ell_{b}=\beta q^{2} / \epsilon$ is the Bjerrum length, $\beta=1 / k_{B} T, q$ is the elementary charge, $\epsilon$ is the dielectric constant, and $\kappa=\sqrt{4 \pi \ell_{b} \sum_{i \neq o} \rho_{i} z_{i}^{2}}$ defines the inverse Debye screening length. From Eq. (1) one can see that, at this level of approximation, the role of the small ions is only to screen the electrostatic interaction between the macroions. ${ }^{1}$

Even though the potential in Eq. (1) is restricted to low concentrations and small colloidal charges, the functional form of the DLVO potential can be extended to describe systems at moderate and high concentrations as well as large colloidal charge. To do this the structural charge $Z_{o}$ is replaced by an effective charge $Z_{\text {eff }}$, which accounts for the nonlinear effects of the counterion condensation. ${ }^{1,5,15-17}$ In fact, it can be formally shown that nonlinear short range correlations within the PM electrolytes can all be introduced into the DH theory by means of appropriate renormalization procedures. ${ }^{18}$ The physical picture behind the charge renormalization is that strong electrostatic attraction between the macroions and the counterions leads to their association, so that from large distances (compared to the Debye length), a macroion can be viewed as carrying charge smaller than its structural bare charge. Both the macroion and its layer of condensed counterions can then be considered as forming a single entity of an effective charge $Z_{\text {eff }}$. Once the nonlinear correlations are taken into account through the charge renormalization, the DLVO pair potential, Eq. (1), can be used in the OCM description to account for the structural properties of colloidal suspensions with monovalent electrolytes.

In this paper, we propose an ansatz which allows us to calculate both the thermodynamic and structural properties of charge stabilized colloidal suspensions in a fully selfconsistent way. This ansatz is based on a coupling of the renormalized jellium model ${ }^{19,20}$ with the OCM OZ integral equations theory. From now on, we will only consider the case of aqueous monovalent electrolytes with $z_{j}=z_{ \pm}= \pm 1$.

\section{THEORETICAL BACKGROUND}

Most of the theoretical work to obtain the effective charge of colloidal particles is based on the mean field Poisson-Boltzmann equation. ${ }^{15}$ In many cases, the infinite dilution limit is employed and the problem reduces to that of a spherical macroion or an infinite planar wall immersed in electrolyte. For a more realistic situation of finite macroion concentration, the colloidal distribution must be incorporated into the PB equation. To do this, one must solve the PB equation for a fixed macroion configuration from which the stress tensor and the force acting on each macroparticle can be calculated. Clearly, a numerical implementation of such procedure is very difficult. ${ }^{9,21}$ To have a more tractable approach, further simplifications are necessary. In this respect two approximations have been proven to be particularly useful: the cell and the renormalized jellium models. Before introducing the new theory, we will make a brief review of the basic features of these approximations and discuss how the effective charges can be extracted from them.

\section{A. Renormalization models}

In the cell model, colloids are assumed to have a quasisolid-state-like structure-macroions arranged in a form of a lattice. This allows us to consider one macroion in a corresponding Wigner-Seitz (WS) cell. A further approximation is to replace the polyhedral WS cell by a cell having the same symmetry as the macroion. ${ }^{22}$ The size of the cell is obtained from the overall macroion concentration. Because of the charge neutrality, the electric field must vanish on the surface of each cell, so that within this approach there is no pair interaction between the colloidal particles. Nevertheless, the model is often used to calculate the effective macroion charges, which enter the DLVO pair interaction potential. To obtain the effective charge, the nonlinear PB equation is solved numerically inside the WS cell. The solution is then asymptotically matched to that of the linearized PB equation with an effective charge-the so called Alexander prescription. $^{22,23}$

The jellium model captures the opposite limit in which the colloid-colloid correlation function is assumed to be completely disordered, ${ }^{11} g_{o o}(r) \approx 1$. This approach is well suited for low colloidal density and weakly charged particles. For strongly charged macroions, the jellium approximation fails to converge. Recently, Trizac and Levin ${ }^{19,20}$ proposed a renormalization procedure designed to extend the validity of the jellium approximation for strongly charged colloidal particles. The renormalized jellium model relies on the concept of counterion condensation to determine the effective charge of the macroions. The method works as follows. One macroion with a charge $Z_{o}$ is positioned at the origin of the coordinate system; the remaining macroions with their condensed counterions are assumed to form a uniform neutralizing background in which the uncondensed counterions and coions move freely. Because it is not know how many counterions will condense onto the colloidal particles, the background charge density is not known a priori, but must be determined self-consistently. The distribution of uncondensed counterions and coion around the central macroion is assumed to be of the Boltzmann form, $g_{o j}(r)=e^{-\beta q z_{j} \psi(r)}$ with $z_{j}= \pm 1$, where $\psi(r)$ is the mean electrostatic potential around the central macroion. The electrostatic potential satisfies the modified Poisson-Boltzmann equation,

$$
\nabla^{2} \psi(r)=-\frac{4 \pi q}{\epsilon}\left(\sum_{j= \pm} \rho_{j} z_{j} e^{-\beta q z_{j} \psi(r)}-Z_{\text {back }} \rho_{\text {back }}(r)\right),
$$

where $Z_{\text {back }}$ and $\rho_{\text {back }}(r)$ are the background charge and density, respectively. In the canonical ensemble-fixed number of all particles $-\rho_{j}$ are determined from the overall electro- 
neutrality, while in the semigrand canonical ensemble, ${ }^{20}$ when the suspension is in contact with a salt reservoir at concentration $c_{s}, \rho_{j}=c_{s}$. We note that Eq. (2) would be exact if the electrostatic potential $\psi(r)$ on the right hand side of Eq. (2) is replaced by the potential of mean force between the microion and colloid, $w(r)$. In that case $Z_{\text {back }}$ would simply be the bare colloidal charge $Z_{o}$ and $\rho_{\text {back }}(r)=\rho_{o} g_{o o}(r)$, where $\rho_{o}$ is the mean colloidal density. Unfortunately, there is no explicit way of calculating the potential of mean force. We are, thus, forced to identify $w(r) \approx \psi(r)$. This is permissible for monovalent ions in aqueous suspensions for which the electrostatic correlations between the microions are small. The price for identifying $w(r) \approx \psi(r)$ is, however, a mandatory renormalization of the colloidal charge. Furthermore, one loses the direct identity between the background density and the colloid-colloid correlation function.

Within the renormalized jellium approximation $\rho_{\text {back }}(r)$ $=\rho_{o}$ and the bulk electroneutrality condition becomes

$$
\sum_{j= \pm} \rho_{j} z_{j} e^{-\beta q z_{j} \psi_{\infty}}-Z_{\text {back }} \rho_{o}=0,
$$

where $\psi_{\infty}$ is the Donnan potential which ensures the overall electroneutrality. In the canonical ensemble, we can take $\psi_{\infty}=0$. For a given set of parameters (including the background charge $\left.Z_{\text {back }}\right)$, Eq. (2) can be solved numerically. Asymptotically, its solution has the form

$$
\psi_{\mathrm{as}}(r)=\psi_{\infty}-\frac{Z_{\mathrm{eff}} q}{\epsilon} \frac{e^{-\kappa(r-a)}}{r(1+\kappa a)} .
$$

In the semigrand canonical ensemble ${ }^{20} \quad \beta q \psi_{\infty}$ $=-\operatorname{arcsinh}\left(Z_{\mathrm{eff}} \rho_{o} / 2 c_{s}\right)$ and the inverse Debye length is $\kappa=\sqrt{8 \pi \ell_{b} c_{s} \cosh \left(\beta q \psi_{\infty}\right)}$. In the canonical ensemble, $\psi_{\infty}=0$ and $\kappa=\sqrt{4 \pi \ell_{b}\left(\rho_{-}+\rho_{+}\right)}$. Equation (4) allows us to calculate the effective charge $Z_{\text {eff }}$ as a function of $Z_{o}$ and $Z_{\text {back }}$. The self-consistency condition is imposed by requiring that $Z_{\text {eff }}$ $=Z_{\text {back }}$, which determines the physical value of the effective colloidal charge. It is important to note that unlike the cell model for which there is no pairwise interaction between the colloids, the macroion-macroion potential of the renormalized jellium model is precisely of the DLVO form.

To extend the renormalized jellium model to larger concentrations, Castañeda-Priego et $a l^{24}$ proposed modifying the uniform background density $\rho_{\text {back }}(r)=\rho_{o}$, to account for the correlation hole around each macroion. These authors observed that, for salt-free suspensions, simulations find that the colloid-colloid correlation function has the first maximum at $r \approx \rho^{-1 / 3}$. They then suggested that this distance can be used to fix the size of the correlation hole between the macroions in salt-free suspensions. ${ }^{24}$ Castañeda-Priego et al. suggested that around each macroion there is an effective exclusion zone of radius $r_{h}=1 / 2 \rho^{1 / 3}$, devoid of the background charge. The factor of 2 is included in order to account for the fact that the exclusion zone is divided equally between the two macroions, see Fig. 1. The exclusion zone around each colloid is then taken into account by replacing the usual uniform jellium background density by a step function $\rho_{\text {back }}(r)=\rho_{o} \Theta\left(r-r_{h}\right)$ in Eq. (2). Such procedure, however, still lacks the self-consistency, since the resulting effec-

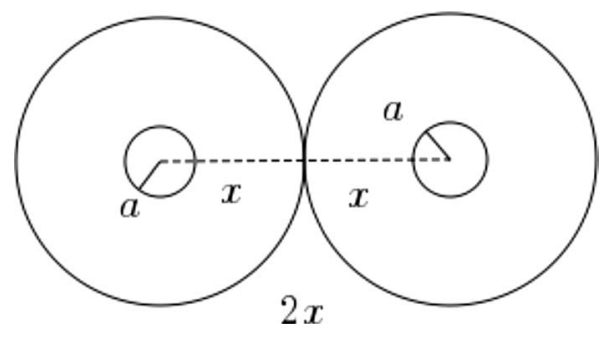

FIG. 1. Two macroions of radius $a$ separated by a distance $r=2 x$, at which the macroion-macroion correlation function has the first maximum. By symmetry, we can define around each macroion an effective exclusion zone of radius $x$.

tive charge cannot be directly related with the correlation function, which is implicit in the form of $\rho_{\text {back }}(r)$. Furthermore, it is not clear how one can extend the above procedure to define the radius of the correlation hole for suspensions containing additional 1:1 electrolyte.

\section{THE SELF-CONSISTENT JELLIUM MODEL}

Although the renormalized jellium model and its modified versions allow us to calculate the effective charges, both theories lack internal self-consistency. In order to correct this, it is necessary to find a way to calculate the effective charge and the correlation function $g_{o o}(r)$ simultaneously. To achieve this, we observe that the background charge in Eq. (2) should be related in some way to the colloid-colloid correlation function. Unfortunately, as discussed above, within the renormalized jellium model, one cannot identify the spatial variation of the background charge directly with the $g_{o o}(r)$. We note, however, that $g_{o o}(r)$ does carry the information about the size of the exclusion zone, which is approximately half the distance to the first peak of $g_{o o}(r)$. In view of this observation, we will make the ansatz of identifying the background density variation with the rescaled colloidcolloid correlation function $\rho_{\text {back }}(r)=\rho_{o} g_{o o}(2 r)$. This choice leads to a uniform background far from colloid $\rho_{\text {back }}(r) \approx \rho_{o}$, while at the same time produces a correlation hole of appropriate size, $\rho_{\text {back }}(r) \approx 0$ for $r<r_{h}$.

With this modification, the fully self-consistent jellium (sc-jellium) equation becomes

$$
\nabla^{2} \phi(\widetilde{r})=-4 \pi \ell_{b} a^{2}\left(\rho_{+} e^{-\phi(\widetilde{r})}-\rho_{-} e^{\phi(\widetilde{r})}\right)+3 \eta \widetilde{Z}_{\text {back }} g_{o o}(2 \widetilde{r})
$$

where we defined the dimensionless quantities $\tilde{r} \equiv r / a$, $\phi(r) \equiv \beta q \psi(r), \widetilde{Z}_{\mathrm{back}} \equiv Z_{\mathrm{back}} \ell_{b} / a$, and $\eta \equiv 4 \pi a^{3} \rho_{o} / 3$. As before, the effective charge is determined by the requirement that $Z_{\text {eff }}=Z_{\text {back }}$. Equation (5) is solved by an iterative procedure. We start with colloid-colloid pair correlation $g_{o o}^{(1)}(r)$ $=1$ and use this in Eq. (5) to extract the corresponding effective and background charge $Z_{\text {eff }}^{(1)}=Z_{\text {back }}^{(1)}$. The system is then considered in the OCM approach, with the interaction potential between the colloids given by 


$$
\beta u(\widetilde{r})=\frac{1}{\Gamma}\left(\frac{\widetilde{Z}_{\mathrm{eff}}}{(1+\kappa a)}\right)^{2} \frac{e^{-\kappa a(\tilde{r}-2)}}{\tilde{r}},
$$

where $\Gamma=\ell_{b} / a$. In the semigrand canonical ensemble, $(\kappa a)^{2}=\left(\kappa_{1} a\right)^{2}\left(1+\left(\kappa_{\text {res }} / \kappa_{1}\right)^{4}\right)^{1 / 2},\left(\kappa_{1} a\right)^{2}=3 \eta \widetilde{Z}_{\text {eff }}$, and $\left(\kappa_{\text {res }} a\right)^{2}$ $=8 \pi \ell_{b} c_{s} a^{2}$. We then numerically solve the one component $\mathrm{OZ}$ integral equation with the Rogers-Young (RY) closure to determine the new pair correlation function $g_{o o}^{(2)}(r)$. This function is then used as a new input in Eq. (5) to calculate the new effective charge $Z_{\text {eff }}^{(2)}$. The procedure is iterated until the convergence is achieved, $g_{o o}^{(i)}(r)=g_{o o}^{(i-1)}(r)$. In practice, only a few iterations are necessary to fulfill this condition.

The RY closure is an interpolation between the hypernetted chain approximation (HNC) and the Percus-Yevick relation, with an adjustable parameter $\alpha$ chosen so as to satisfy the thermodynamic self-consistency in the calculation of the isothermal compressibility. ${ }^{25}$ In the salt-free case, the major contribution to the osmotic pressure comes from counterions, so that $\alpha$ is determined by imposing the requirement that

$$
\frac{\partial(\beta P)}{\partial \rho}=1+\rho \hat{h}_{o o}(0) \approx \frac{\tilde{Z}_{\mathrm{eff}}}{\Gamma},
$$

where $\hat{h}_{o o}$ is the Fourier transform of the total correlation function. The first equality is the Kirkwood-Buff relation, ${ }^{26}$ while the second one comes from approximating the microion pressure by the jellium equation of state, ${ }^{19,20} \beta P=Z_{\text {eff }} \rho$, and disregarding the weak dependence of the effective charge on the macroion density.

For the case of large salt concentrations and moderate volume fractions-when the density dependence of the effective pair potential is weak - the pressure is given by that of the OCM, ${ }^{27,28}$ and the last equality in Eq. (7) is replaced by the OCM inverse compressibility,

$$
1+\rho \hat{h}_{o o}(0)=\frac{\partial\left(\beta P_{\mathrm{OCM}}\right)}{\partial \rho} .
$$

The OCM pressure $P_{\mathrm{OCM}}$ can be calculated from the pair correlation function using the well known virial equation. It is important to note that, in calculating the right hand side of Eq. (8), the interaction potential must be kept constant. ${ }^{27} \mathrm{We}$ use Eq. (8) to determine $\alpha$ in the RY closure when dealing with suspensions in contact with a salt reservoir at large concentration.

In practice, due to finite discretization, the calculated correlation function is not equal to 1 for large distances. Instead, it oscillates around 1 with a small amplitude. This creates difficulty for the numerical solution of the PB equation. In order to ensure the correct long-distance behavior of $\psi(r)$, we set a cutoff distance $\widetilde{r}_{c}$, beyond which we force $g_{o o}(\widetilde{r})=1$. The value of $\widetilde{r}_{c}$ is chosen such that $\left|g_{o o}(\widetilde{r})-1\right|$ $<0.0025$ for $\tilde{r}>\widetilde{r}_{c}$.

\section{RESULTS}

In Fig. 2 we plot the macroion-macroion correlation functions calculated using the sc-jellium model developed above and compare it with the results of the modified jellium (m-jellium) approximation of Castañeda-Priego et al. and
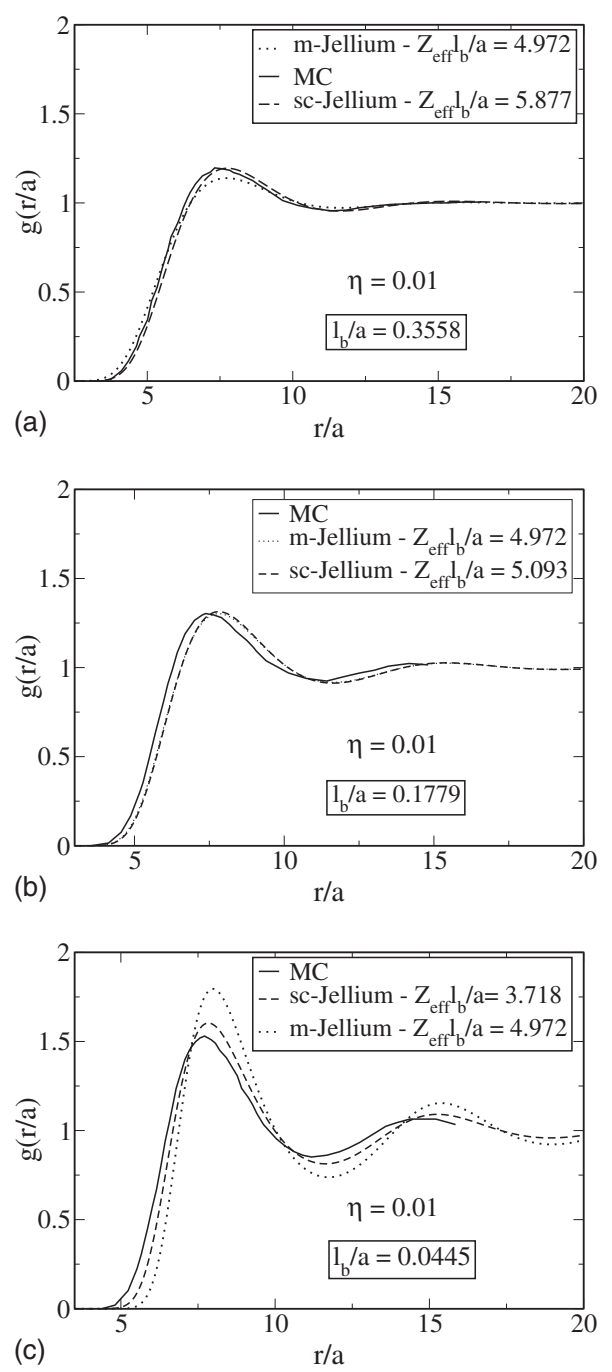

FIG. 2. Macroion-macroion correlation functions calculated using the scjellium (dashed lines), m-jellium (dotted lines), and MC simulations (Ref. 29) (solid lines) for a de-ionized colloidal suspensions at volume fraction $\eta=0.01$. The coupling parameters are (a) $\Gamma=0.3558$, (b) $\Gamma=0.1779$, and (c) $\Gamma=0.0445$.

with the MC simulations performed by Linse ${ }^{29}$ for aqueous de-ionized suspension, $\ell_{b} \approx 7.2 \AA, \kappa_{\text {res }}=0$ and colloidal volume fraction $\eta=0.01$. Three systems with coupling parameters $\Gamma=0.3558$ (a), 0.1779 (b), and 0.0445 (c), corresponding to particles of radius $a \approx 160,40$, and $20 \AA$, respectively, were studied. All the calculations were performed in the saturation limit (very large bare charge). The corresponding effective charges are displayed in the graphs. As can be seen, both the sc-jellium approach and the m-jellium show good agreement with the MC simulations for $\Gamma=0.3558$ and $\Gamma$ $=0.1779$, while for the lowest value $\Gamma=0.0445$ the $\mathrm{m}$-jellium model seems to strongly overestimate the colloidal structure.

In Fig. 3 we compare the correlation functions calculated using the sc-jellium with the MC simulations for various colloidal volume fractions at fixed coupling parameter $\Gamma$ $=0.3558$ in the no-salt regime. Again, a good agreement with the MC simulations is found for all the macroion concentrations. Surprisingly, this agreement seems to be better at higher volume fractions, diminishing as the concentration becomes very low. Figure 4 shows the behavior of the effec- 


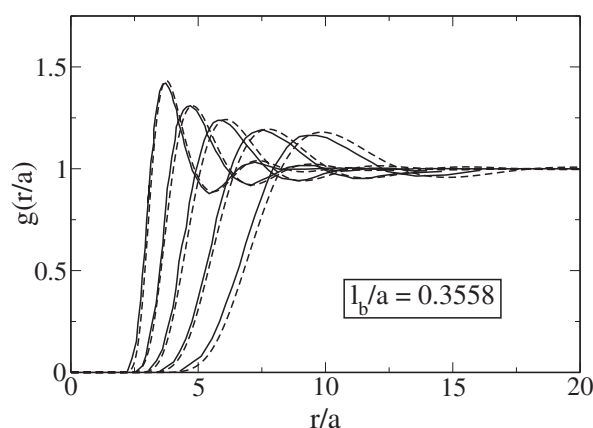

FIG. 3. Macroion-macroion correlation functions calculated using the scjellium (dashed lines) and MC simulations (Ref. 29) (solid lines) for a deionized colloidal suspension with coupling parameter $\Gamma=0.3558$. From left to right, the volume fractions are $\eta=0.08, \eta=0.04, \eta=0.02, \eta=0.01$, and $\eta=0.005$.

tive charge as a function of the colloidal volume fraction for the sc-jellium (dashed curve), m-jellium (dotted curve), and the original renormalized jellium model of Trizac and Levin (solid curve). Although the qualitative behavior is the same for all three models, there is a significant quantitative variation in the value of the effective charge. The effective charges predicted by the sc-jellium lie between those of the $\mathrm{m}$-jellium and the renormalized jellium models.

The real advantage of the sc-jellium over the m-jellium is that it allows us to accurately calculate the effective charges and structures for suspensions containing 1:1 electrolyte. At the moment this is the only theory capable of doing this for strongly charged colloidal particles. The effects of nonzero salt concentration on the macroion structure can be seen in Fig. 5, where the correlation functions for reservoir salt concentrations corresponding to $\kappa_{\text {res }} a=1.0$ and $\kappa_{\text {res }} a=1.5$ are displayed for various volume fractions. In Fig. 6 , the predictions of the theory are compared with the results of MC simulations performed by Lobaskin and Qamhieh ${ }^{30}$ for aqueous suspensions containing monovalent salt. Once again, the calculated correlation functions are found to be in good agreement with MC simulations.

In the case of suspensions containing 1:1 electrolyte, we see some very general trends. As expected, increase in salt concentration leads to larger screening and loss of colloidal structure. In the salt dominated regime, the correlation functions become nearly independent of the macroion concentra-

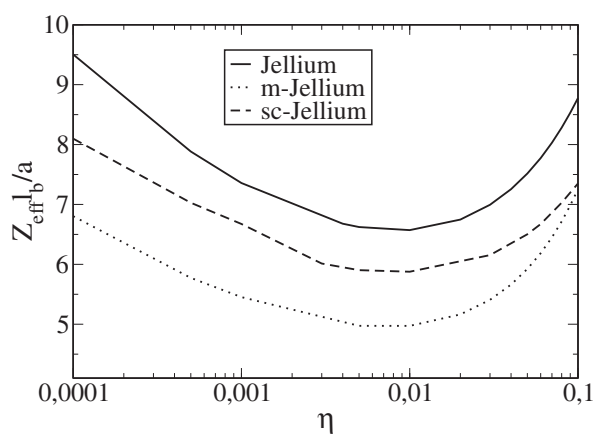

FIG. 4. Reduced effective charge as a function of volume fraction for a de-ionized colloidal suspension with $\Gamma=0.3558$, as predicted by the renormalized jellium model (solid curve), m-jellium (dotted curve) and the fully self-consistent approach developed in this paper (dashed curve).
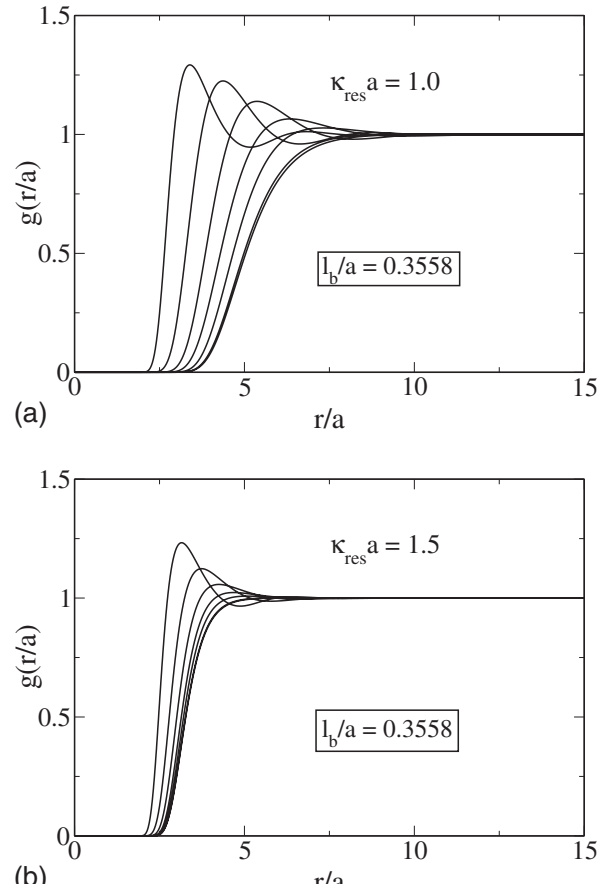

FIG. 5. Macroion-macroion correlation functions calculated using the scjellium, for suspension with $\Gamma=0.3558$ in contact with a salt reservoir at $\kappa_{\text {res }} a=1.0$ in (a) and $\kappa_{\text {res }} a=1.5$ in (b). From left to right, colloidal volume fractions are $\eta=0.08, \eta=0.04, \eta=0.02, \eta=0.01, \eta=0.005, \eta=0.001$, and $\eta=0.0005$. As the volume fraction decreases, the correlation functions become nearly independent on the macroion concentration and the curves start to overlap.

tion. For these cases, both the effective potential and the effective colloidal charge show very slow variation with the colloidal volume fraction; this also explains the weak variation of the correlation functions. Another remarkable feature is that, at high salt concentrations, the colloidal structure is no longer important for the computation of the effective charge, Fig. 7. The effective charge calculated using the original renormalized jellium model with a uniform background and the sc-jellium are practically the same.

\section{SUMMARY AND CONCLUSIONS}

We developed a theory that allows us to self-consistently calculate both the thermodynamic and structural properties

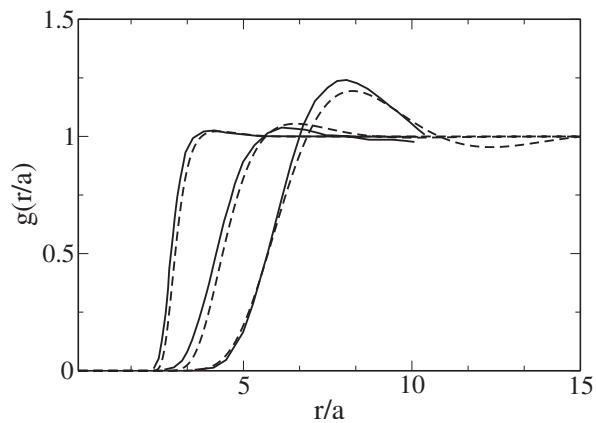

FIG. 6. Colloid-colloid pair correlation functions for an aqueous suspension with added 1:1 electrolyte at room temperature and volume fraction $\eta$ $=0.0084$. Colloidal radius is $a=20 \AA$. The continuous line are from MC simulations (Ref. 30), while the dashed lines are the predictions of the sc-jellium model. From left to right, the reservoir screening lengths are given by $\kappa_{\text {res }} a=3.28, \kappa_{\text {res }} a=1.04$, and $\kappa_{\text {res }} a=0$. The corresponding effective charges predicted by the sc-jellium approach are $\widetilde{Z}_{\text {eff }}=19.5, \widetilde{Z}_{\text {eff }}=9.22$, and $\widetilde{Z}_{\text {eff }}=6.05$, respectively. 


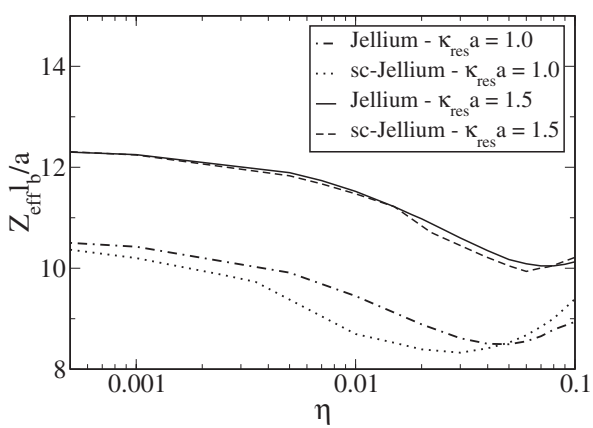

FIG. 7. Reduced effective charge as a function of the volume fraction as predicted by the renormalized jellium and the sc-jellium models.

of aqueous colloidal suspensions containing 1:1 electrolyte. The theory is based on coupling of the OZ equation with RY closure to the PB equation with the renormalized jellium approximation. The predictions of the theory were compared to the MC simulations and were found to be in good agreement. Finally, it is important to stress that the theory developed above applies only to aqueous suspensions containing monovalent counterions. In this case the correlations between the microions are small and the interaction potential between the colloidal particles is well approximated by the DLVO potential. This will no longer be the case for suspensions containing multivalent counterions or in solvents of low dielectric constant. Under these conditions one finds many other interesting effects, such as like-charge attraction and the reversal of the electrophoretic mobility. There is an extensive literature dealing with these fascinating phenomena. ${ }^{1,31}$

\section{ACKNOWLEDGMENTS}

T.E.C. would like to acknowledge useful conversations with S. Pianegonda during the early stages of this work. This research is supported in part by CNPq, INCT-FCx of Brazil, and by the Air Force Office of Scientific Research (AFOSR), USA, under Grant No. FA9550-09-1-0283.

${ }^{1}$ Y. Levin, Rep. Prog. Phys. 65, 1577 (2002).

${ }^{2}$ L. Belloni, J. Phys.: Condens. Matter 12, R549 (2000).

${ }^{3}$ H. Boroudjerdi, Y. W. Kim, A. Naji, R. R. Netz, X. Schlagberger, and A. Serr, Phys. Rep. 416, 129 (2005).

${ }^{4}$ R. Messina, J. Phys.: Condens. Matter 21, 113102 (2009).

${ }^{5}$ M. Quesada-Pérez, J. Callejas-Fernández, and R. Hidalgo-Álvarez, Adv. Colloid Interface Sci. 95, 295 (2002).

${ }^{6}$ A.-P. Hynninen and A. Z. Panagiotopoulos, Phys. Rev. Lett. 98, 198301 (2007); Mol. Phys. 106, 2039 (2008); A. J. Banchio, M. G. McPhie, and G. P. Nagele, J. Phys.: Condens. Matter 20, 404213 (2008); A. J. Banchio and G. P. Nagele, J. Chem. Phys. 128, 104903 (2008); D. Bratko, T. Cellmer, J. M. Prausnitz, and H. W. Blanch, Biotechnol. Bioeng. 96, 1 (2007); P. Linse, Adv. Polym. Sci. 185, 111 (2005).

${ }^{7}$ Y. Levin, M. C. Barbosa, and M. N. Tamashiro, Europhys. Lett. 41, 123
(1998); R. van Roij, M. Dijkstra, and J. P. Hansen, Phys. Rev. E 59, 2010 (1999); P. B. Warren, J. Chem. Phys. 112, 4683 (2000); A. Diehl, M. C. Barbosa, and Y. Levin, Europhys. Lett. 53, 86 (2001); Y. Levin, E. Trizac, and L. Bocquet, J. Phys.: Condens. Matter 15, S3523 (2003); A. Denton, Phys. Rev. E 76, 051401 (2007).

${ }^{8}$ A. V. Brukhno, T. Akesson, and B. Jönsson, J. Phys. Chem. B 113, 6766 (2009); V. Lobaskin, A. Lyubartsev, and P. Linse, Phys. Rev. E 63, 020401 (2001); E. Allahyarov, E. Zaccarelli, F. Sciortino, P. Tartaglia, and H. Lowen, Europhys. Lett. 78, 38002 (2007).

${ }^{9}$ J. Dobnikar, Y. Chen, R. Rzehak, and H. H. von Grünberg, J. Chem. Phys. 119, 4971 (2003).

${ }^{10}$ L. Belloni, J. Chem. Phys. 85, 519 (1986).

${ }^{11}$ B. Beresford-Smith, D. Y. C. Chan, and D. J. Mitchell, J. Colloid Interface Sci. 105, 216 (1985).

${ }^{12}$ J.-P Hansen and H. Löwen, Annu. Rev. Phys. Chem. 51, 209 (2000).

${ }^{13}$ P. González-Mozuelos and M. D. Carbajal-Tinoco, J. Chem. Phys. 109, 11074 (1998).

${ }^{14}$ A. R. Denton, Phys. Rev. E 70, 031404 (2004).

${ }^{15}$ L. Belloni, Colloids Surf., A 140, 227 (1998).

${ }^{16}$ L. Shapran, H. J. Schöpe, and T. Palberg, J. Chem. Phys. 125, 194714 (2006).

${ }^{17}$ G. S. Manning, J. Chem. Phys. 51, 924 (1969); Y. Levin, Europhys. Lett. 34, 405 (1996); Y. Levin and M. C. Barbosa, J. Phys. II 7, 37 (1997); P. S. Kuhn, Y. Levin, and M. C. Barbosa, Macromolecules 31, 8347 (1998).

${ }^{18}$ R. Kjellander and D. J. Mitchell, J. Chem. Phys. 101, 603 (1994).

${ }^{19}$ E. Trizac and Y. Levin, Phys. Rev. E 69, 031403 (2004).

${ }^{20}$ S. Pianegonda, E. Trizac, and Y. Levin, J. Chem. Phys. 126, 014702 (2007).

${ }^{21}$ J. Dobnikar, D. Halozan, M. Brumen, H. H. von Grünberg, and R. Rzehak, Comput. Phys. Commun. 159, 73 (2004).

${ }^{22}$ S. Alexander, P. M. Chaikin, P. Grant, G. J. Morales, P. Pincus, and D. Hone, J. Chem. Phys. 80, 5776 (1984).

${ }^{23}$ E. Trizac, M. Aubouy, L. Bocquet, and H. H. von Grünberg, Langmuir 19, 4027 (2003).

${ }^{24}$ R. Castañeda-Priego, L. F. Rojas-Ochoa, V. Lobaskin, and J. C. MixtecoSánchez, Phys. Rev. E 74, 051408 (2006).

${ }^{25}$ F. J. Rogers and D. A. Young, Phys. Rev. A 30, 999 (1984).

${ }^{26}$ J.-P. Hansen and I. R. McDonald, Theory of Simple Liquids, 2nd ed. (Academic, London, 1986).

${ }^{27}$ E. Trizac, J. Dobnikar, L. Belloni, H. H. von Grünberg, and R. Castañeda-Priego, Phys. Rev. E 75, 011401 (2007).

${ }^{28}$ J. Dobnikar, R. Castañeda-Priego, H. H. von Grünberg, and E. Trizac, New J. Phys. 8, 277 (2006).

${ }^{29}$ P. Linse, J. Chem. Phys. 113, 4359 (2000).

${ }^{30}$ V. Lobaskin and K. Qamhieh, J. Phys. Chem. B 107, 8022 (2003).

${ }^{31}$ G. N. Patey, J. Chem. Phys. 72, 5763 (1980); R. Kjellander and S. Marcelja, J. Phys. Chem. 90, 1230 (1986); N. Grønbech-Jensen, R. J. Mashl, R. F. Bruinsma, and W. M. Gelbart, Phys. Rev. Lett. 78, 2477 (1997); E. Allahyarov, I. D'Amico, and H. Lowen, ibid. 81, 1334 (1998); J. J. Arenzon, J. F. Stilck, and Y. Levin, Eur. Phys. J. B 12, 79 (1999); A. Diehl, H. A. Carmona, and Y. Levin, Phys. Rev. E 64, 011804 (2001); A. W. C. Lau, P. Pincus, D. Levine, and H. A. Fertig, ibid. 63, 051604 (2001); J. Z. Wu, D. Bratko, H. W. Blanch, and J. M. Prausnitz, J. Chem. Phys. 111, 7084 (1999); F. J. Solis and M. O. de la Cruz, Phys. Rev. E 60, 4496 (1999); M. Tanaka and A. Y. Grosberg, J. Chem. Phys. 115, 567 (2001); F. J. Solis and M. O. de la Cruz, Eur. Phys. J. E 4, 143 (2001); A. Y. Grosberg, T. T. Nguyen, and B. I. Shklovskii, Rev. Mod. Phys. 74, 329 (2002); A. Martin-Molina, M. Quesada-Perez, F. GalisteoGonzalez, and R. Hidalgo-Alvarez, J. Phys.: Condens. Matter 15, S3475 (2003); F. W. Tavares, D. Bratko, and J. M. Prausnitz, J. Phys. Chem. B 108, 9228 (2004); A. A. Kornyshev, D. J. Lee, S. Leikin, and A. Wynveen, Rev. Mod. Phys. 79, 943 (2007); A. Diehl and Y. Levin, J. Chem. Phys. 129, 124506 (2008). 